\title{
Intermediate-age Galactic open clusters: fundamental parameters of NGC 2627
}

\author{
Andrés E. Piatti, ${ }^{1 \star}$ Juan J. Clariáa ${ }^{2 \star}$ and Andrea V. Ahumada ${ }^{2 \star}$ \\ ${ }^{1}$ Instituto de Astronomía y Física del Espacio, CC 67, Suc. 28, 1428 Capital Federal, Argentina \\ ${ }^{2}$ Observatorio Astronómico, Laprida 854, 5000 Córdoba, Argentina
}

Accepted 2003 August 1. Received 2003 July 22; in original form 2003 June 10

\begin{abstract}
Charge-coupled device (CCD) photometry in the Johnson $V$, Kron-Cousins $I$ and Washington $C M T_{1}$ systems is presented in the field of the poorly known open cluster NGC 2627 . Four independent Washington abundance indices yield a mean cluster metallicity of $[\mathrm{Fe} / \mathrm{H}]=$ $-0.12 \pm 0.08$, which is compatible with the existence of a radial gradient in the Galactic disc. The resultant colour-magnitude diagrams indicate that the cluster is an intermediate-age object of $1.4 \mathrm{Gyr}$. Based on the best fits of the Geneva group's isochrones to the $(V, V-I)$ and $\left(T_{1}\right.$, $\left.C-T_{1}\right)$ diagrams, we estimate $E(V-I)=0.25 \pm 0.05$ and $V-M_{V}=11.80 \pm 0.25$ for $\log t=9.15$, and $E\left(C-T_{1}\right)=0.23 \pm 0.07$ and $T_{1}-M_{T_{1}}=11.85 \pm 0.25$ for $\log t=9.10$, respectively, assuming solar metal content. The derived reddening value $E\left(C-T_{1}\right)$ implies $E(B-V)=0.12 \pm 0.07$ and a distance from the Sun of $2.0 \pm 0.4 \mathrm{kpc}$. Using the WEBDA data base and the available literature, we re-examined the overall properties of all the open clusters with ages between 0.6 and $2.5 \mathrm{Gyr}$. We identified peaks of cluster formation at $0.7-0.8$, $1.0-1.1,1.6-1.7$ and $2.0-2.1 \mathrm{Gyr}$, separated by relative quiescent epochs of $\sim 0.2-0.3 \mathrm{Gyr}$. We also estimated a radial abundance gradient of $-0.08 \pm 0.02$, which is consistent with the most recent determinations for the Galactic disc, but no clear evidence for a gradient perpendicular to the Galactic plane is found.
\end{abstract}

Key words: techniques: photometric - open clusters and associations: general - open clusters and associations: individual: NGC 2627.

\section{INTRODUCTION}

Intermediate-age and old Galactic open clusters are located in the disc of the Galaxy and are very important as probes of both age and metallicity in the dynamical and chemical evolution of the Galactic disc. In particular, the oldest clusters place constraints upon the age of the disc, whereas the distributions shown in metallicity, age and space trace the subsequent evolution of the disc. Investigations focusing on possible abundance gradients in the disc and the expected age-metallicity parameters (e.g. Friel \& Janes 1993; Chen, Hou \& Wang 2003) require data on the greatest possible number of intermediate-age and old open clusters.

The present study is part of an ongoing project of observation of open clusters located in different regions of the Milky Way. We intend to obtain good-quality photometry in the Johnson $V$, KronCousins $I\left(V I_{\mathrm{KC}}\right)$ and Washington $C M T_{1}$ systems, in order that we may not only enlarge the sample of studied open clusters but also estimate their fundamental parameters accurately.

^E-mail: andres@iafe.uba.ar (AEP); claria@mail.oac.uncor.edu (JJC); andrea@mail.oac.uncor.edu (AVA)
NGC 2627 (IAU designation C0835-297), also known as Mel 87 (Melotte 1915), Cr 188 (Collinder 1931), BH 38 (van den Bergh \& Hagen 1975) or ESO 431SC20 (Lauberts 1982), is located in a moderately rich star field in Pyxis at $\alpha_{2000}=8^{\mathrm{h}} 37^{\mathrm{m}} \cdot 2, \delta_{2000}=-29^{\circ} 56^{\prime} .9$ and $l=251.58, b=+6.65$. Ruprecht (1966) considered it to belong to Trumpler (1930) class III $2 \mathrm{~m}$, i.e. a moderately populated open cluster with no noticeable concentration and a medium range in the brightness of the stars.

The presence of several red giant candidates in the cluster field makes this cluster interesting for a morphological study of the red giant region in relation to previous results (Clariá, Mermilliod \& Piatti 1999; Mermilliod et al. 2001; Clariá et al. 2003). In addition, NGC 2627 is in itself worth a detailed study because no previous Washington photometry has been obtained.

It is somewhat surprising that such a comparatively bright and apparently little reddened open cluster has been the subject of relatively few previous studies. NGC 2627 was included by King (1964) in a list of clusters that appeared to be old on the basis of their appearance on the Palomar Observatory Sky Survey (POSS) prints. However, according to the charge-coupled device (CCD) $U B V I_{\mathrm{C}}$ photometry carried out in the innermost cluster area by Ramsay \& Pollaco (1992), NGC 2627 appears to be younger than 300 Myr. These 
authors also derived a mean colour excess $E(B-V)=0.63 \pm 0.04$ and a distance from the Sun of $3.31 \mathrm{kpc}$. Soon afterwards, in an extensive CCD photometric survey of potential old open clusters, Phelps, Janes \& Montgomery (1994) obtained CCD VI photometry up to $V \sim 19.5$ in the cluster field. They defined the morphological age index $\delta V$ as the magnitude difference between the mainsequence turnoff and the clump in the $(V, V-I)$ colour-magnitude diagram (CMD), deriving $\delta V=1.6$ for NGC 2627. This value implies a cluster age of $2.8 \mathrm{Gyr}$ (Janes \& Phelps 1994), thus estimating the cluster to be considerably older than Ramsay \& Pollaco thought. Janes \& Phelps (1994) derived a distance of $1.91 \mathrm{kpc}$ by relating the cluster's angular diameter taken from Lyngå (1987) to an assumed value for the linear diameter. Ahumada (1995) carried out a CCD UBVRI photometric study of NGC 2627, deriving the following very preliminary cluster parameters from the fitting of Meynet, Mermilliod \& Maeder's (1993) isochrones: $\langle E(B-V)\rangle \sim 0.05$ and $\log ($ age $) \sim 9.4$ (equivalent to $2.5 \mathrm{Gyr}$ ). The latter value shows very good agreement with the one previously estimated by Phelps et al. (1994). Note, however, the substantial difference between the mean colour excesses derived by Ramsay \& Pollaco (1992) and Ahumada (1995). More recently, Dutra \& Bica (2000) derived a far-infrared reddening $E(B-V)_{\mathrm{FIR}}=0.15$ for NGC 2627 from DIRBE/IRAS $100 \mu \mathrm{m}$ dust emission in the cluster region. Although this far-infrared reddening agrees well with that derived using the extinction maps built by Burstein \& Heiles (1982), the aforementioned works prove that there is no strong agreement on the parameters for NGC 2627, as derived by various studies. Furthermore, we believe that this lack of coherence warrants a redetermination of such parameters, based on data of superior reliability.

In this paper we report the results obtained from $C C D V I_{\mathrm{KC}}$ photometry up to $V \sim 19.5$ and CCD photometry in the $C, M$ and $T_{1}$ bands of the Washington system up to $\sim 18.5$ in the cluster area. These data are used to make new and independent determinations of reddening, distance, age and metallicity of NGC 2627 . We decided to use the Washington system because of its combination of broad bands and high metallicity sensitivity provided by the $C$ filter, and the wide colour baseline between $C$ and $T_{1}$. The advantages of the Washington system in deriving abundances in $\mathrm{G}$ and/or $\mathrm{K}$ giants of open clusters have already been carefully identified by Geisler, Clariá \& Minniti (1991).

In Section 2 we describe the observational material and the data reduction. In Section 3 we present the analysis of the photometric data, while in Section 4 we determine the cluster parameters (reddening, distance, age and metallicity). The results here obtained for NGC 2627, together with those known for 54 open clusters with ages between 0.6 and $2.5 \mathrm{Gyr}$, are discussed in Section 5. A brief summary of our conclusions is offered in Section 6.

\section{OBSERVATIONS AND REDUCTIONS}

We obtained CCD images of the cluster field with the Johnson $V$, Kron-Cousins $I$ and Washington $C, M$ and $T_{1}$ filters, using the $0.9-\mathrm{m}$ telescope at the Cerro Tololo Inter-American Observatory (CTIO), Chile, on 1997 December 23-24. Note that the standard Washington $\mathrm{T}_{2}$ filter has long been known to be identical to the Kron-Cousins $I$ filter (Canterna 1976; Bessell 1979). The recommended prescription for the $C$ filter that we used is the one given in Geisler (1996): $3 \mathrm{~mm}$ $\mathrm{BG} 3+2 \mathrm{~mm}$ BG40, although Geisler (private communication) now recommends the following prescription: $3 \mathrm{~mm} \mathrm{BG3}+4 \mathrm{~mm} \mathrm{BG} 40$, the significant advantage of which lies in the avoidance of the small red leak present in the previous prescription.
The observations were carried out with the $2048 \times 2048$ Tektronix 2K \#3, as described in (Piatti, Clariá \& Ahumada 2003, hereafter Paper I) for the open cluster NGC 2194. The scale on the chip is $0.40 \operatorname{arcsec}$ pixel $^{-1}$ (focal ratio $f / 13.5$ ), yielding an area of $13.6 \times$ $13.6 \mathrm{arcmin}^{2}$. The CCD was controlled by the CTIO ARCON 3.3 data acquisition system in the standard quad amplifier mode operating at a gain setting of $5 \mathrm{e}^{-} / \mathrm{ADU}$ with a readout noise of $4 \mathrm{e}^{-}$. We obtained two 10-s and one 20-s $V$ exposures, two 5-s and one 10-s $I$ exposures, two 30-s $C$ exposures, two 10 -s $M$ exposures, and two 5-s $T_{1}$ exposures for NGC 2627.

Fig. 1 shows a schematic finding chart of the cluster field built with all the measured stars in the $V$ band. In the present work, as in Paper I, we calibrated the observations in the $V I_{\mathrm{KC}}$ and Washington systems through the observation of numerous standard stars from the lists of Landolt (1992) and Geisler (1996). The data were reduced at the Instituto de Astronomía y Física del Espacio with a stand-alone version of DAOPHOT II (Stetson 1987), after trimming, bias subtraction and flat-fielding. More details on the reduction and calibration procedures are given in Paper I.

Table 1 gives in succession a running star number of the observed stars, $X$ and $Y$ coordinates in pixels (Fig. 1), $V$ magnitudes and $V-I$ colours, together with the corresponding observational errors $\sigma(V)$ and $\sigma(V-I)$, the number of observations and the star identification given by Phelps et al. (1994). This table was built as explained in Paper I. (Only a portion of this table is shown here for guidance regarding its form and content, its whole content being available in the on-line version of the journal on Synergy.) In the same way, we built Table 2 (also available in full on Synergy), including the $T_{1}$ magnitudes and $C-T_{1}$, colours for all the measured stars, together with the $X$ and $Y$ coordinates and the corresponding photometric errors. $M-T_{1}, T_{1}-T_{2}$ and $C-M$ colours are provided in Table 3 for red giant candidates.

The calculated internal accuracy of the photometry has been computed according to the criteria given by Stetson (1987) and includes random noise, errors in the modelling and centring of the stellar profile. These internal standard errors provided by DAOPHOT for the $V$ magnitude and $V-I$ colours have been plotted against their corresponding visual magnitudes in Fig. 2. Fig. 3 shows how the differences between our $V$ magnitudes and those obtained by Phelps et al. (1994) vary as a function of $V$. Offsets of $\Delta\left(V_{\text {our }}-V_{\text {PJM }}\right)$ $=-0.022 \pm 0.112$ and $\Delta\left[(V-I)_{\mathrm{our}}-(V-I)_{\mathrm{PJM}}\right]=-0.051 \pm$ 0.107 have been computed from 491 stars measured in common. As mentioned explicitly by Phelps et al. (1994), some of their observing was done under marginal conditions, which could explain not only the relatively large offset in $V$, but also the scatter seen in the upper panel of Fig. 3. Unfortunately, it is just not possible to compare our VI photometry with that of Ahumada (1995) due to the fact that this author did not publish his magnitudes and colours.

\section{ANALYSIS OF THE DATA}

\subsection{The colour-magnitude diagrams}

Fig. 4 depicts the resulting $(V, V-I)$ colour-magnitude diagram (CMD) obtained using all the measured stars. The most relevant features are an upper main sequence (MS) with a remarkable hooking envelope, and a dirty broad lower MS indicating the possible existence of field contamination. Indeed, two distinct sequences appear in the CMD from $V \sim 17$ towards fainter magnitudes. On the one hand, the upper cluster MS follows along redder colours and tends to disappear beyond $V \sim 18$, where a break in its curvature is also 


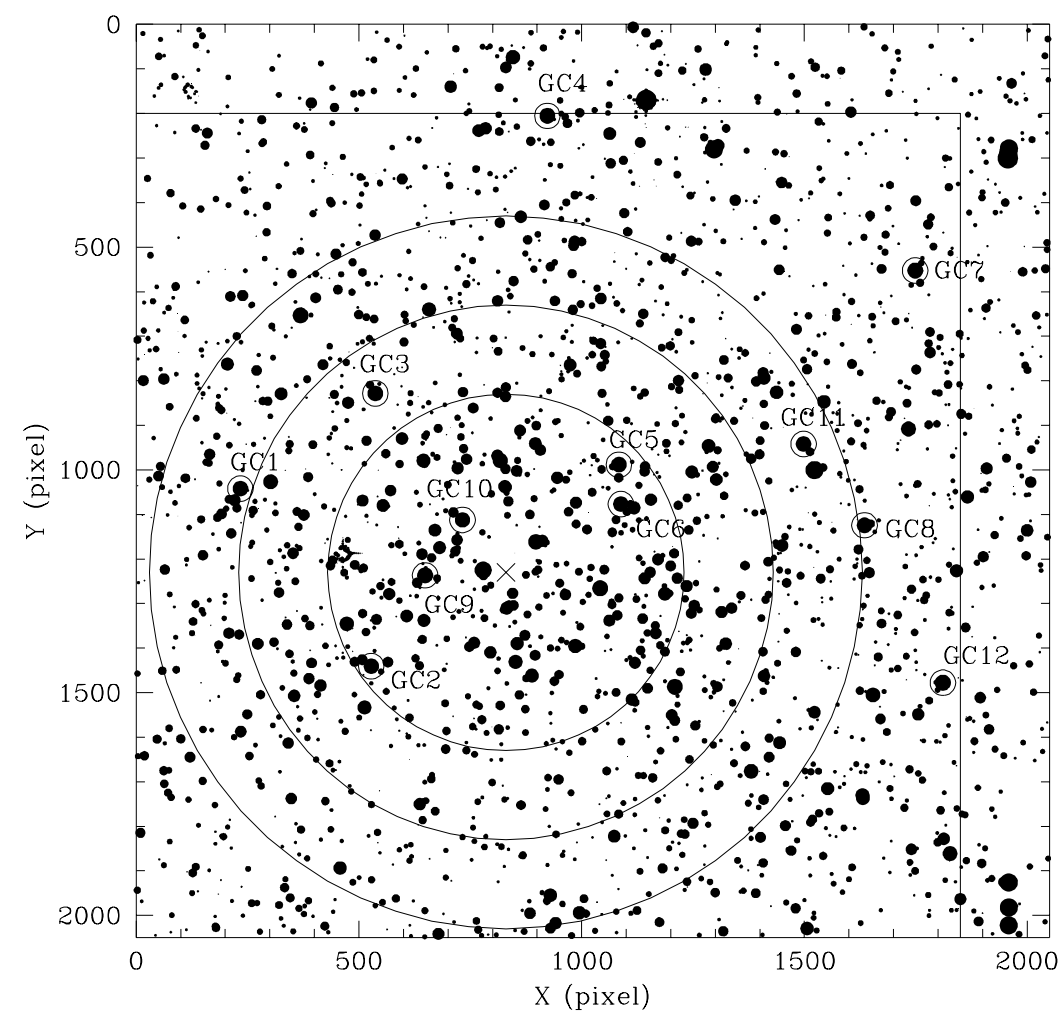

Figure 1. Schematic finding chart of the stars observed in the field of NGC 2627. North is up and east is to the left. The sizes of the plotting symbols are proportional to the $V$ brightness of the stars. The 12 bright giant candidates discussed in Section 4.2 are identified.

Table 1. CCD VI data of stars in the field of NGC 2627. ${ }^{a}$

\begin{tabular}{lcccccccc}
\hline Star & $\begin{array}{c}X \\
\text { (pixel) }\end{array}$ & $\begin{array}{c}Y \\
\text { (pixel) }\end{array}$ & $\begin{array}{c}V \\
(\mathrm{mag})\end{array}$ & $\begin{array}{c}\sigma(V) \\
(\mathrm{mag})\end{array}$ & $\begin{array}{c}V-I \\
(\mathrm{mag})\end{array}$ & $\begin{array}{c}\sigma(V-I) \\
(\mathrm{mag})\end{array}$ & $n$ & ID PJM94 $^{b}$ \\
\hline 1 & 1633.887 & 5.652 & 16.973 & 0.021 & 0.867 & 0.042 & 1 & \\
2 & 1115.442 & 6.589 & 14.413 & 0.008 & 0.645 & 0.023 & 2 & \\
3 & 1631.044 & 8.164 & 19.953 & 0.227 & 0.968 & 0.439 & 1 & \\
4 & 1856.501 & 9.087 & 19.808 & 0.099 & 1.812 & 0.142 & 1 & \\
5 & 141.281 & 12.213 & 17.988 & 0.045 & 1.434 & 0.060 & 3 & \\
$\vdots$ & $\vdots$ & $\vdots$ & $\vdots$ & $\vdots$ & $\vdots$ & $\vdots$ & $\vdots$ &
\end{tabular}

$\bar{a}$ Only part of this table is shown for guidance as to its form and content. The whole table is available from: http://www.blackwellpublishing.com/ products/journals/suppmat/MNR/MNR7061/MNR7061sm.htm

${ }^{b}$ The last column lists the star identification given by Phelps et al. (1994).

produced and, on the other hand, there is a much more crowded, twice or three times broader, bluer star sequence. The morphology and location of this latter sequence indicates the existence of field stars located along the cluster line of sight mainly belonging to the old MS disc population. Another interesting feature of the $(V, V-$ I) $\mathrm{CMD}$ is the presence of a number of good candidates for giant clump (GC) stars centred at $(V, V-I) \sim(11.9,1.13)$. As we do not know the radial velocities, we cannot assess membership of these stars. However, since half of them lie within 2.5 arcmin from the cluster centre (Fig. 1), we may reasonably expect many of them to be cluster giants.

To investigate whether the broadness and scatter of the cluster MS is not originated by errors in our photometry, we inspected the number of measures per star and their uncertainties. Our data show that 60 per cent of the total number of measured stars have three measures of their $V$ magnitudes and $V-I$ colours and approxi-
Table 2. CCD $C T_{1}$ data of stars in the field of NGC 2627.

\begin{tabular}{lccccccc}
\hline Star & $\begin{array}{c}X \\
\text { (pixel) }\end{array}$ & $\begin{array}{c}Y \\
\text { (pixel) }\end{array}$ & $\begin{array}{c}T_{1} \\
(\mathrm{mag})\end{array}$ & $\begin{array}{c}\sigma\left(T_{1}\right) \\
(\mathrm{mag})\end{array}$ & $\begin{array}{c}C-T_{1} \\
(\mathrm{mag})\end{array}$ & $\begin{array}{c}\sigma\left(C-T_{1}\right) \\
(\mathrm{mag})\end{array}$ & $n$ \\
\hline 10 & 2045.767 & 32.818 & 16.728 & 0.044 & 1.003 & 0.014 & 2 \\
11 & 55.288 & 34.270 & 17.662 & 0.191 & 1.343 & 0.200 & 1 \\
11 & 1874.580 & 34.877 & 18.079 & 0.165 & 1.313 & 0.182 & 1 \\
$\vdots$ & $\vdots$ & $\vdots$ & $\vdots$ & $\vdots$ & $\vdots$ & $\vdots$ & $\vdots$
\end{tabular}

${ }^{a}$ Only part of this table is shown for guidance as to its form and content. The whole table is available from: http://www.blackwellpublishing.com/ products/journals/suppmat/MNR/MNR7061/MNR7061sm.htm

mately expand from the brightest limit down to $V=19$, while 15 and 25 per cent of the measured stars have two and one measures and cover $V$ ranges from 14 to 20 mag and from 19 until the photometric limit, respectively. Thus, the photometric errors of stars with three measures are mainly those shown in Fig. 2, i.e. $\sigma_{V} \leqslant 0.05 \mathrm{mag}$ for $V=19$ and $\approx 0.01$ for $10<V<15$, and $\sigma_{V-I} \leqslant 0.01 \mathrm{mag}$ for $V=13$ and 0.05 for $V \leqslant 17$. According to this census, the cluster features apparently remain unaltered when Fig. 4 is constructed using only stars with photometric uncertainties within 0.01 mag from the mean error at any $V$ magnitude level. In the subsequent analysis we use stars with three or two measures, since those with only one measure are not significant for the cluster study.

Since the contamination by field stars can disturb the drawing of a clear fiducial cluster MS, we evaluated such a possibility in the cluster CMD by first determining the cluster centre, and then building CMDs of stars distributed in different circular extractions centred on the cluster. To determine the central coordinates of the cluster, we built projected stellar density profiles along the $X$ and $Y$ directions. The histograms with the cumulative number of stars 


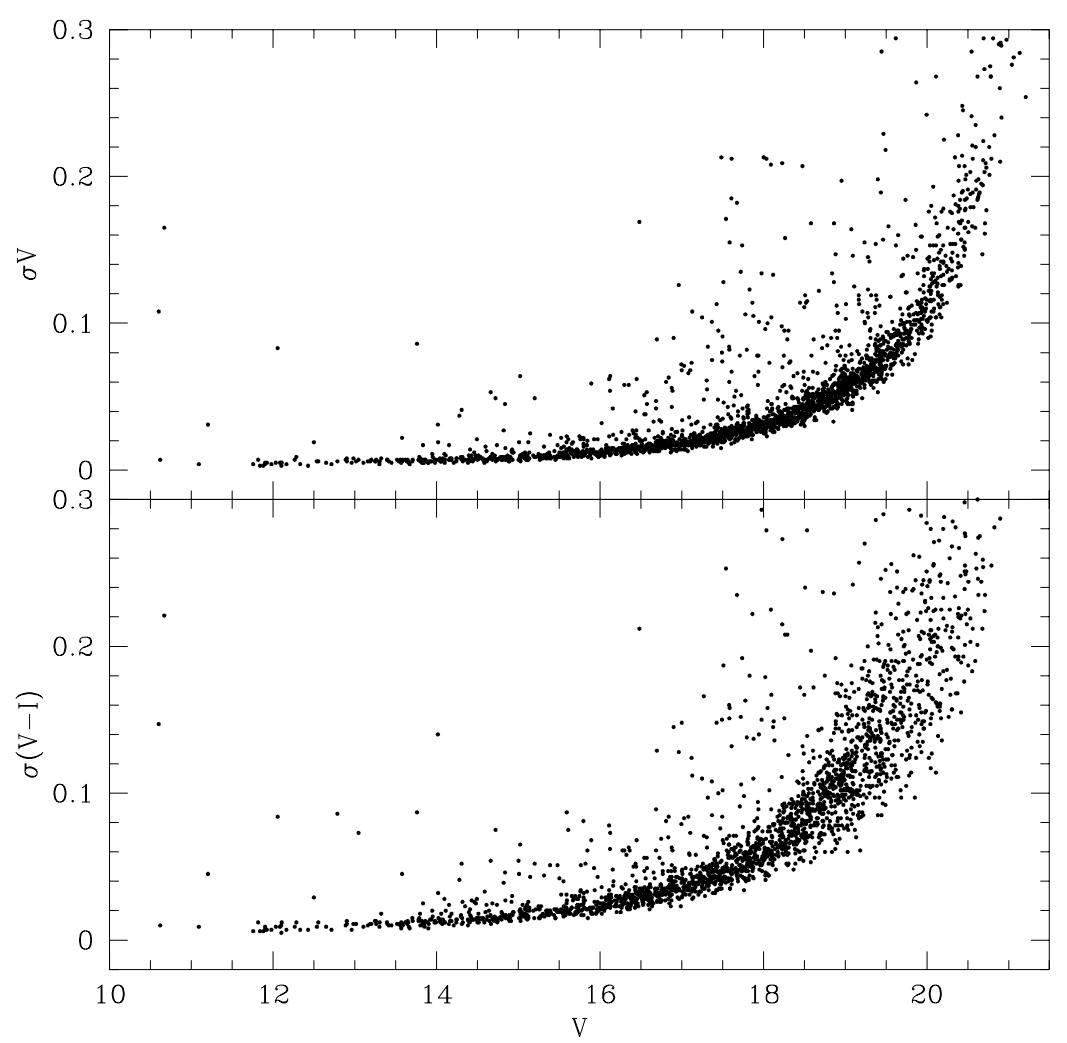

Figure 2. Magnitude and colour photometric errors as a function of $V$.

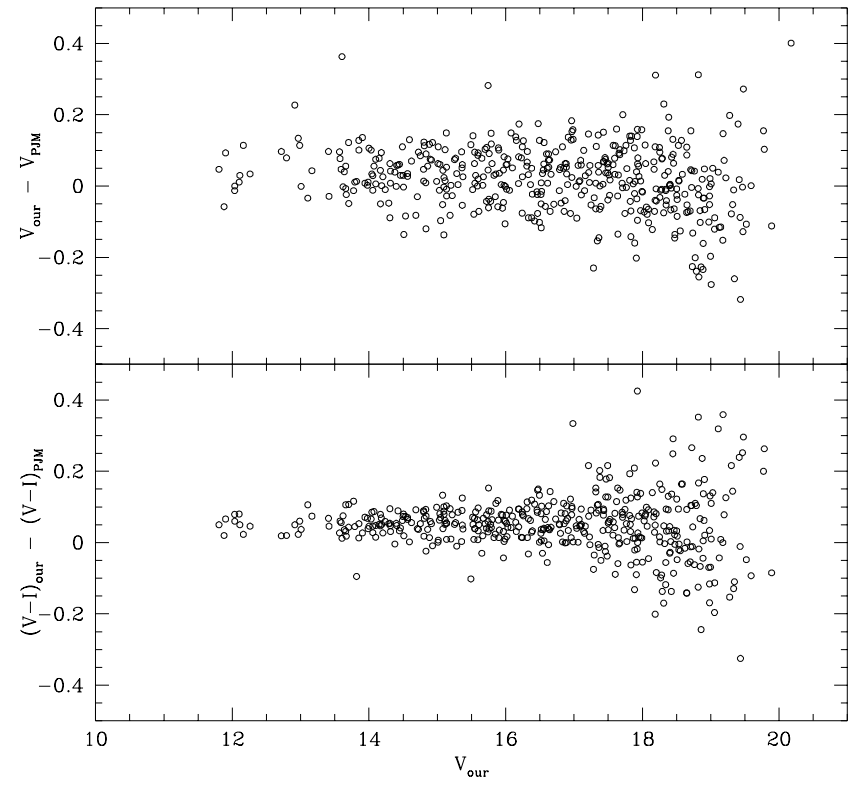

Figure 3. Differences between our $V$ magnitudes and those obtained by Phelps et al. (1994) as a function of $V$.

counted in both directions were built using coordinate intervals of 30 pixels. The selected bin size allowed us to sample the spatial star distribution statistically and avoid spurious peaks that mimic central cluster regions due to the high resolution of the images and the presence of groups of stars spreading out throughout the field. No more detailed stellar profiles are obtained using a narrower bin size, but additional noise, which increases as the coordinate intervals get smaller. We used the finding chart of Fig. 1 for checking the position

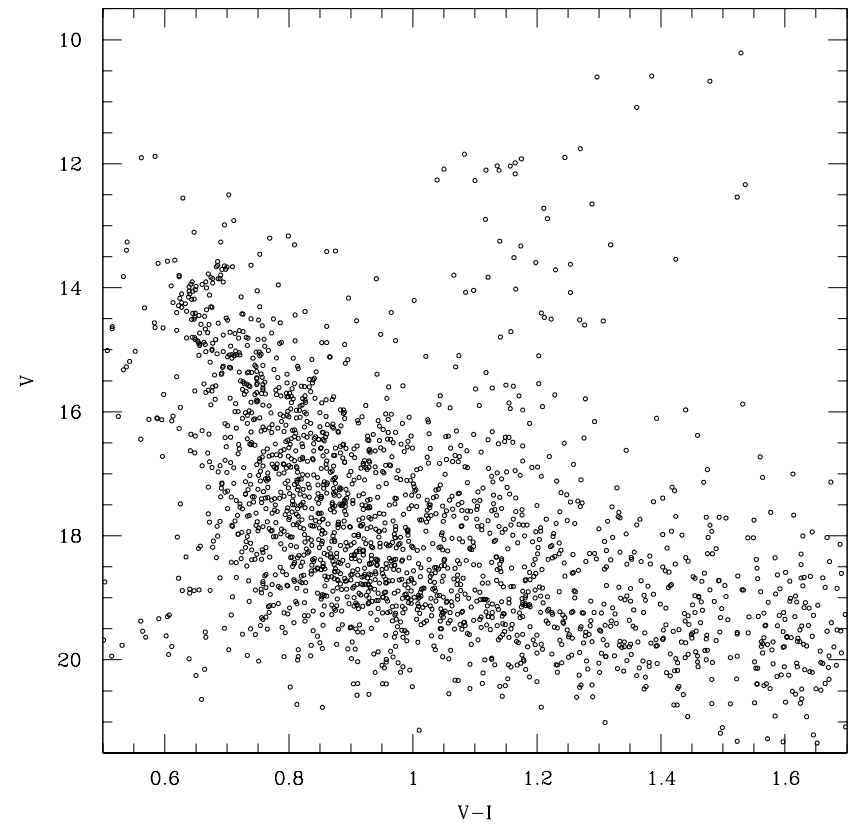

Figure 4. $(V, V-I)$ colour-magnitude diagram for stars observed in the field of NGC 2627.

of the cluster centre obtained by this method. Finally, we adopted the position $\left(X_{\mathrm{C}}, Y_{\mathrm{C}}\right)=(830,1230)$ pixel as the cluster centre, which is indicated in Fig. 1 with an $X$ symbol.

The task of selecting the sizes of the circular extractions was not as straightforward as desired. The choice of the radii for different circular extractions was an iterative, somewhat subjective process 
designed to obtain the best representation of the cluster and its transition to the field. For this purpose, we used as reference the full width at half-maximum of the radial stellar density distribution. Fig. 1 shows the circular extractions adopted with radii of 400 , 600 and 800 pixel, respectively. The radius of the smallest circular extraction was fixed to guarantee the presence of a predominant number of cluster stars over field stars in the extracted CMD. The bigger circular extractions were used to obtain a better definition of the fiducial cluster sequence from a larger number of cluster stars. Although cluster stars could be distributed throughout the entire observed field, the chance of finding them dominating along the field edges over field stars is negligible. For this reason, we defined the surrounding field extraction as the region encompassed by the two fringes 200 pixels wide located along the upper and right margins of Fig. 1.

The resulting $r<400$ pixel CMD is shown in the upper-left panel of Fig. 5. It reveals the main cluster features, i.e. the upper MS with a populated hooking turnoff (TO) and the GC. On the other hand, the upper-right panel presents the CMD, which is representative of the cluster surrounding field. Note that most of these stars lie towards fainter magnitudes and an important percentage of them are concentrated towards bluer colours. The different features between the cluster core $(r<400$ pixel) and field CMDs are remarkable. Thus, by using the upper-right panel as the surrounding cluster field $\mathrm{CMD}$, we applied a cleaning process to the bigger circular extraction
CMDs in order to recover as many observed cluster stars as possible. First, we divided the field CMD into boxes of 0.5 and 0.05 mag in $V$ and $V-I$, respectively. We then counted the number of stars in each box and subtracted from the circular extraction CMDs a number of stars $\left(N_{i, j}\right)$ given by the expression:

$N_{i, j}=\left(A_{i} / A_{\mathrm{f}}\right) N_{\mathrm{f}, j}$,

where $N_{\mathrm{f}, j}$ is the number of field stars counted in the $j$ th box and $A_{\mathrm{f}}$ and $A_{i}$ represent the areas covered by the surrounding field and circular extraction, respectively. The method assumes a uniform distribution over the entire field of stars of any spectral type. In Fig. 5, the lower panels illustrate the performance of this method, and show the $r<600$ pixel CMD before and after applying the cleaning process. As can be seen, most of the field stellar population was satisfactorily removed. For the $r<800$ pixel CMD we obtained a similar result, although the residuals are more important. Therefore, with the aim of estimating the cluster fundamental parameters, the cleaned $r<$ 600 pixel CMD was preferred, since a reasonable balance between maximizing the number of stars necessary to define the fiducial cluster sequence and minimizing the contamination of field stars was achieved.

We treated the Washington data following the same scheme of analysis as for the $V, I$ photometry. Fig. 6 shows the $\left(T_{1}, C-T_{1}\right)$ $\mathrm{CMD}$ for all the measured stars with three or two measures. The observed colour range became twice as large as that of the $(V, V-I)$
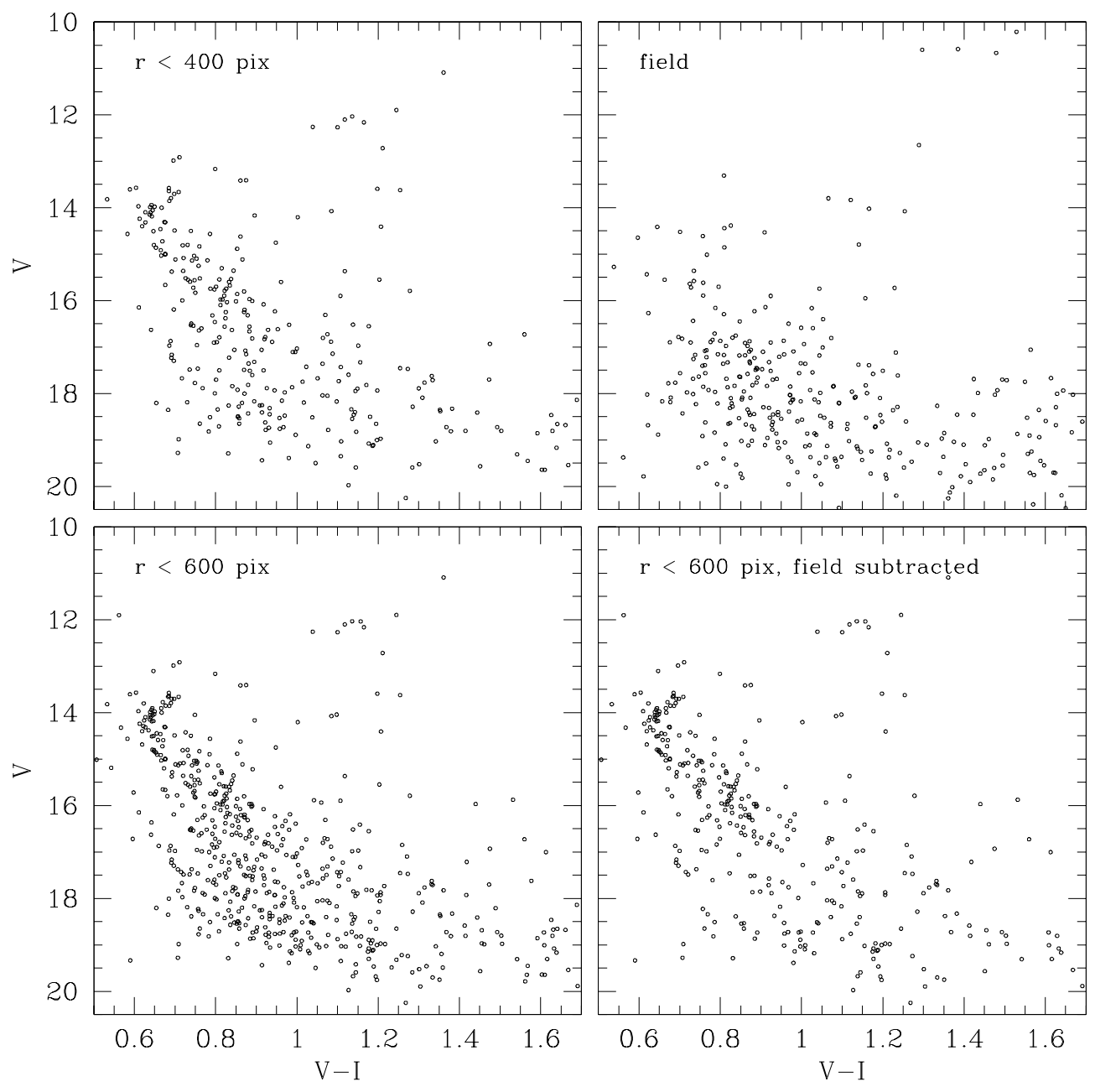

Figure 5. $(V, V-I)$ colour-magnitude diagram for stars observed in different extracted regions as indicated in each panel. See Section 3 for details. 


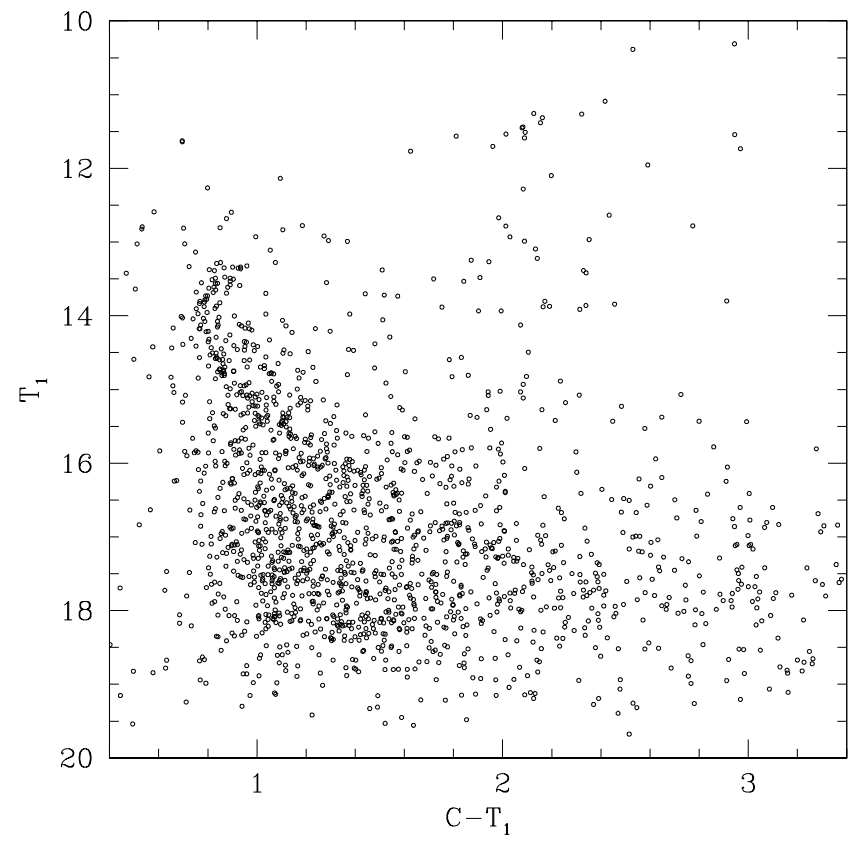

Figure 6. $\left(T_{1}, C-T_{1}\right)$ colour-magnitude diagram for stars observed in the field of NGC 2627.
CMD, which in turn leads to some subtle differences: (i) the cluster MS appears more clearly defined and without any visible break at fainter magnitudes, its lower and upper envelopes appearing better outlined and separated from field stars; and (ii) the TO presents a smoother curvature. The star field cleaning process was performed following the same precepts as for the $(V, V-I) \mathrm{CMD}$, except for the colour width of the boxes, which were fixed to 0.1 mag.

\subsection{Binary stars}

A secondary star sequence is clearly seen not only in the cluster core region $\left(T_{1}, C-T_{1}\right)$ CMD of Fig. $7(r<400$ pixel), but also in the field subtracted $r<600$ pixel CMD. This evidence is less convincing when looking at the $(V, V-I) \mathrm{CMD}$, although it is still discernible. This secondary sequence is formed by stars lying above the cluster MS, at a distance of $\sim 0.6-0.7$ mag, which is perfectly predictable in the presence of a significant fraction of binary stars. As is well known, the occurrence of binary stars in open clusters is not a rare phenomenon (see e.g. Mermilliod \& Mayor 1989, 1990). The quite distinct secondary sequence clearly seen in the $(V, V-I)$ and $\left(T_{1}\right.$, $C-T_{1}$ ) CMDs, discernible even through the field contamination, suggests the more than likely presence of a sizeable population of binary stars $(\geqslant 20$ per cent) in NGC 2627 .
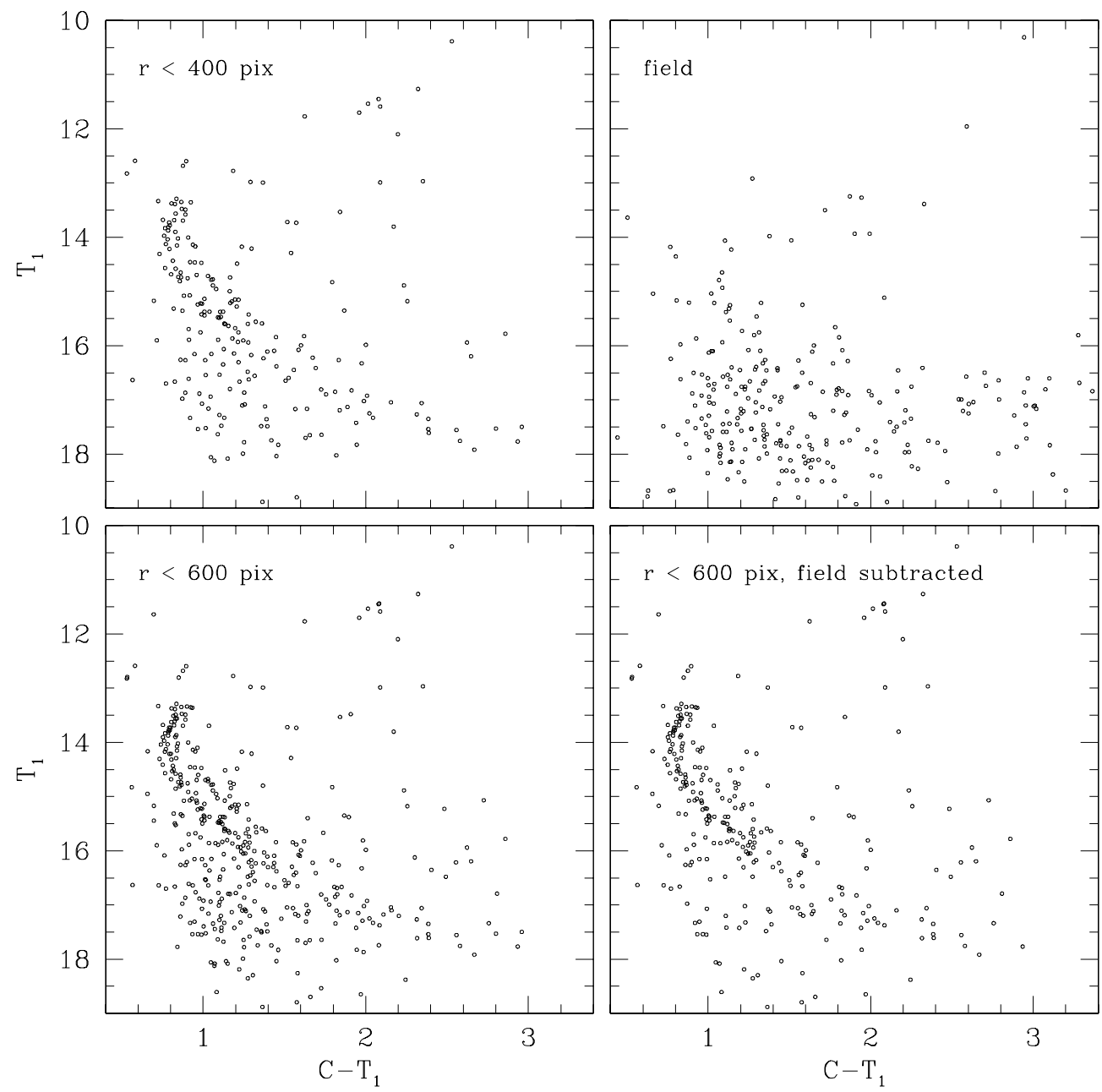

Figure 7. $\left(T_{1}, C-T_{1}\right)$ colour-magnitude diagram for stars observed in different extracted regions as indicated in each panel. See Section 3 for details. 


\section{CLUSTER FUNDAMENTAL PARAMETERS}

\subsection{Reddening, distance and age}

To estimate the $E(V-I)$ colour excess, the $V-M_{V}$ apparent distance modulus, the age and the metallicity of NGC 2627, we fitted theoretical isochrones computed by Lejeune \& Schaerer (2001, hereafter LS01) to the observed $(V, V-I)$ CMD. The isochrones, which cover the age range from $10^{3} \mathrm{yr}$ to $16-20 \mathrm{Gyr}$ in steps of $\Delta \log t=0.05$ dex, were calculated for the entire set of non-rotating Geneva stellar evolution models, covering masses from $0.4-0.8$ to $120-150 \mathrm{M}_{\odot}$ and metallicities from $Z=0.0004$ to 0.1 . To select a subsample of isochrones, we used as reference the age value derived from the $\delta V$ index (Phelps et al. 1994). We measured $\delta V=$ $1.0 \pm 0.1$, which corresponds to an age of $1.5 \pm 0.2$ Gyr (Janes \& Phelps 1994). When selecting subsets of isochrones for different $Z$ values to address the metallicity effect in the cluster fundamental parameters, we preferred those including overshooting effect.

Regarding the fit, we took advantage of the clearly shaped cluster MS and focused particularly on the TO morphology. We thus built a double-parametric table with two entries listing the $E(V-I)$ colour excess and $V-M_{V}$ apparent distance modulus derived for each combination of age and metallicity. The $E(V-I)$ and $V-M_{V}$ values used in the fit of the isochrone that most resembles the cluster fiducial MS were adopted as the cluster reddening and apparent distance modulus. We finally derived $E(V-I)=0.25 \pm 0.05$ and $V-M_{V}=$ $11.80 \pm 0.25$ for $\log t=9.15$ and $Z=0.02$. The uncertainties in the derived parameters arise from the propagation of the estimated age dispersion ( $\Delta t=200 \mathrm{Myr})$ due to a random combination of age and metallicity. We adopted a solar metal content for the cluster, since isochrones for $Z=0.008$ and 0.04 yield fits either with fainter or bluer TOs. In addition, isochrones for both metal abundances have subgiant branches developed along slightly more different colour intervals from those of the isochrones computed for solar metallicity.

Fig. 8 shows the isochrone that most resembles the cluster features with a solid line, and two additional isochrones with dotted and dashed lines to illustrate the combination of varying age and metallicity in the fitting procedure. Notice that the MS is satisfactorily fitted within the errors by the isochrone corresponding to $\log t=$ 9.15. The latter also appears to tightly fit possible cluster stars placed in the transition path from the MS to the GC. However, the predicted position of the GC is $0.10-0.15$ mag redder and almost 1 mag fainter than the observed GC. Neither from the good agreement found between the ages derived from the $\delta V$ index, which takes into account the placement of the GC and the TO, nor from the isochrones fitting, did we find any explanation for the difference, unless it could arise from the input physics and evolutionary codes used. Similar findings have been obtained in other open clusters (see e.g. Clariá et al. 1994). By using 1.33 and 3.2 for the ratios $E(V-I) / E(B-V)$ and $A_{V} / E(B-V)$ (Cousins 1978), we obtained $E(B-V)=0.19 \pm$ 0.05 and $V_{\mathrm{o}}-M_{V}=11.2 \pm 0.3$, which implies a distance from the Sun of $1.7 \pm 0.4 \mathrm{kpc}$. The distance error was computed with the expression $\sigma(d)=0.46\left[\sigma\left(V-M_{V}\right)+3.2 \sigma(E(B-V))\right] d$, where $\sigma\left(V-M_{V}\right)$ and $\sigma(E(B-V))$ represent the estimated errors in $V-M_{V}$ and $E(B-V)$, respectively.

The Washington photometry offers the alternative of probing the resulting cluster parameters from a direct independent estimate of them by fitting isochrones in the $\left(T_{1}, C-T_{1}\right)$ CMD as described above for the $(V, V-I)$ CMD. Fig. 9 depicts a summary of the results obtained using the theoretical isochrones calculated by LS01 for the Washington system for different metallicity values. Dashed, solid and dotted lines represent isochrones for $\log t=9.00,9.10$ and 9.20

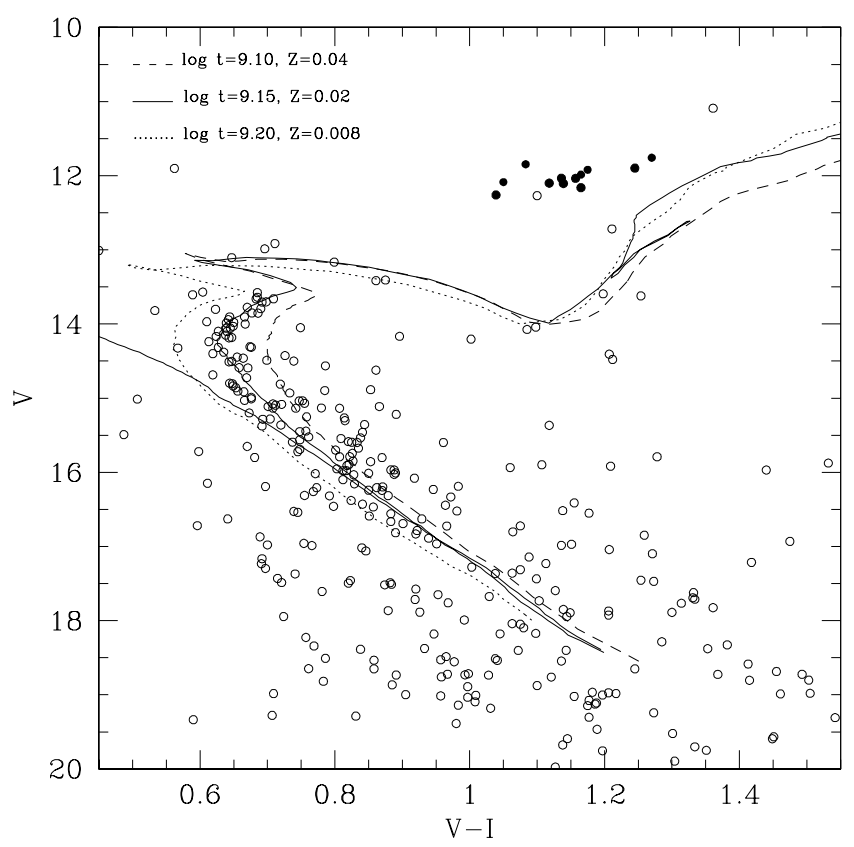

Figure 8. $(V, V-I)$ CMDs for stars in NGC 2627. Isochrones from Lejeune \& Schaerer (2001), computed taking overshooting into account, are overplotted. Filled circles represent giant candidates discussed in Section 4.2.

and $Z=0.04,0.02$ and 0.008 , respectively. The best fit is achieved for the isochrone of $\log t=9.10$ and solar metal content. From this fit, we derive $E\left(C-T_{1}\right)=0.23 \pm 0.07$ and $T_{1}-M_{T_{1}}=11.85 \pm$ 0.25 . By using the $E\left(C-T_{1}\right) / E(B-V)$ and $A_{T_{1}} / E(B-V)$ ratios given by Geisler, Lee $\& \operatorname{Kim}(1996)$, we obtained $E(B-V)=$ $0.12 \pm 0.07$ and $V_{\mathrm{o}}-M_{V}=11.5 \pm 0.4$ (equivalent to $2.0 \pm$ $0.4 \mathrm{kpc}$ ). These values, which show good agreement within $1 \sigma$ with those arising from the $V, I$ data, have been adopted in the present study.

\subsection{Metal content}

We estimated the mean cluster metal abundance by measuring five Washington metallicity-sensitive indices, previously calibrated in terms of high-dispersion spectroscopic iron-to-hydrogen ratios by Geisler et al. (1991, hereafter GCM91) for late-type stars. According to the authors, the metal content of a $\mathrm{G}-\mathrm{K}$ giant star can be easily obtained through an iterative method they propose, although the accuracy of the resulting metallicity value strongly depends on the quality of the photometric data. Typically, a precision of the order of $\sim 0.01$ is required in the Washington colour indices. Therefore, for the stars observed in the field of NGC 2627, we applied the criterion suggested in Paper I, wherein we estimated the mean metal abundance of the open cluster NGC 2194. Thus, we conservatively keep for the analysis those stars that verify $m_{f}(i=14)$ $-m_{f}(i=15)<0.01 \mathrm{mag}$, where $m_{f}(i)$ is the instrumental magnitude of a star measured through the passband $f$ with aperture of $i$ pixels in radius. Among these stars we in turn selected giant candidates with $T_{1}<12$ and $C-T_{1}>1.5$ (see Fig. 6). All the selected giant candidates turned out to be point spread function (PSF) stars, i.e. bright and well-isolated stellar objects placed in free-bad pixel CCD regions. In addition, although the stand-alone version of ALLSTAR II provides reliable PSF magnitudes, we instead preferred the aperture magnitudes measured for the selected stars, since the former can be affected by residuals of the multiprofile fit performed by ALLSTAR to all the stars in the frames simultaneously. Table 3 lists $X$ and $Y$ coordinates in pixels, $T_{1}$ magnitudes, $C-T_{1}$, 


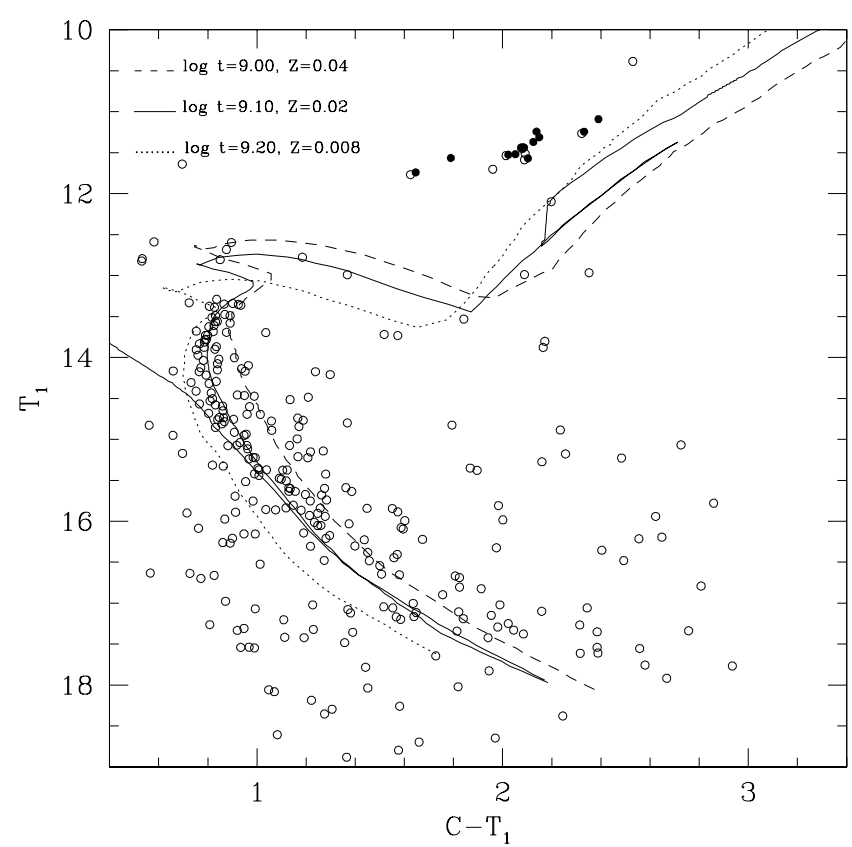

Figure 9. $\left(T_{1}, C-T_{1}\right)$ CMDs for stars in NGC 2627. Isochrones from Lejeune \& Schaerer (2001), computed taking overshooting into account, are overplotted. Filled circles represent red giant candidates discussed in Section 4.2.

$M-T_{1}$ and $T_{1}-T_{2}$ colours, and the corresponding observational errors for only 12 bright giant candidates, which satisfy the established requirement. The last column lists the number of individual observations.

To determine the mean cluster metallicity following the precepts outlined by GCM91, we have adopted $E(B-V)=0.12$ for NGC 2627. Specifically, we use the reddening-corrected iso-abundance relations between the indices $C-M$ and $T_{1}-T_{2}, C-T_{1}$ and $T_{1}-$ $T_{2}, C-M$ and $M-T_{2}$, and $C-T_{1}$ and $M-T_{2}$. We have not employed the iso-abundance relation between $M-T_{1}$ and $T_{1}-T_{2}$ on account of the fact that the resulting abundances in this case are not consistent with the ones obtained using the other four relations, an outcome for which we are unable to offer an explanation. Following GCM91's nomenclature, we denote $\Delta^{\prime}(C-M)_{T_{1}-T_{2}}$ as $\Delta_{1}^{\prime}$, $\Delta^{\prime}\left(C-T_{1}\right)_{T_{1}-T_{2}}$ as $\Delta_{3}^{\prime}, \Delta^{\prime}(C-M)_{M-T_{2}}$ as $\Delta_{4}^{\prime}$ and $\Delta^{\prime}\left(C-T_{1}\right)_{M-T_{2}}$ as $\Delta_{5}^{\prime}$. For each cluster red giant candidate, we determine the above four metallicity indices, using the reddening ratios given by GCM91 for the Washington system.

Fig. 10 displays the four unreddened Washington colour-colour diagrams, wherein iso-abundance lines for $[\mathrm{Fe} / \mathrm{H}]=+0.5,0.0$, $-0.5,-1.0$ and -1.5 have been plotted. The Washington abundance indices calculated for the above 12 red giant candidates are presented in columns (2)-(6) of Table 4. Stars 9, 10, 11 and 12 (open circles in Fig. 10) fall outside the cluster clump so that they are not relevant to the derivation of mean cluster metallicity. The resulting mean values and standard deviations for the remaining eight red clump stars are: $\Delta_{1}^{\prime}=0.007 \pm 0.015, \Delta_{3}^{\prime}=0.063 \pm 0.033, \Delta_{4}^{\prime}=$ $-0.075 \pm 0.005$ and $\Delta_{5}^{\prime}=-0.060 \pm 0.011$, which imply $[\mathrm{Fe} / \mathrm{H}]_{1}=$ $0.04 \pm 0.04,[\mathrm{Fe} / \mathrm{H}]_{3}=0.14 \pm 0.06,[\mathrm{Fe} / \mathrm{H}]_{4}=-0.27 \pm 0.02$ and $[\mathrm{Fe} / \mathrm{H}]_{5}=-0.18 \pm 0.04$, if the calibrations of GCM91 are used. As shown by GCM91, the $M-T_{2}$ colour provides a much broader baseline than the $T_{1}-T_{2}$ colour and is thus much less susceptible to photometric errors in determining abundances. Therefore, the two best indices for deriving metallicities in virtually all cases are $\Delta_{4}^{\prime}$ and $\Delta_{5}^{\prime}$. This is why we averaged the four $[\mathrm{Fe} / \mathrm{H}]$ values by assigning double weight to the $[\mathrm{Fe} / \mathrm{H}]_{4}$ and $[\mathrm{Fe} / \mathrm{H}]_{5}$ values. We therefore adopted for NGC 2627 a weighted mean of the four abundance determinations, i.e. $[\mathrm{Fe} / \mathrm{H}]=-0.12 \pm 0.08\left(\sigma_{\mathrm{p}}\right)$, where $\sigma_{\mathrm{p}}$ is the standard deviation of the mean. We are aware of the somewhat large metallicity range derived from the different Washington abundance indices, even though we took care to select the highest-quality photometric data. Since the Washington filters gather information from different, relatively wide spectral regions, we have reached the conclusion that the uncertainty estimated for the cluster metallicity may have its origin in a combination of intrinsic anomalies of some heavy elements and the limiting precision of the broad-band photometry. For this reason, we have preferred to adopt a weighted average of the four Washington abundance estimates in order to have a measure of the overall cluster metallicity.

The derived cluster metal content places NGC 2627 in the metalpoor side of the distribution of intermediate-age and old open clusters. Note that the derived metallicity practically does not change if stars 9, 10, 11 and 12 (open circles in Fig. 10) are included in the mean, since in this case the resulting metallicity turns out to be $[\mathrm{Fe} / \mathrm{H}]=-0.16 \pm 0.08\left(\sigma_{\mathrm{p}}\right)$.

\section{DISCUSSION}

Ramsay \& Pollaco (1992) computed individual reddening values using the expression $E(U-B) / E(B-V)=0.72+0.05 E(B-V)$,

Table 3. CCD Washington photometry of 12 red giant candidates in the field of NGC 2627.

\begin{tabular}{|c|c|c|c|c|c|c|c|c|c|c|c|}
\hline Star & $\begin{array}{c}X \\
\text { (pixel) }\end{array}$ & $\begin{array}{c}Y \\
\text { (pixel) }\end{array}$ & $\begin{array}{c}T_{1} \\
(\mathrm{mag})\end{array}$ & $\begin{array}{l}\sigma\left(T_{1}\right) \\
(\mathrm{mag})\end{array}$ & $\begin{array}{c}C-T_{1} \\
(\mathrm{mag})\end{array}$ & $\begin{array}{c}\sigma\left(C-T_{1}\right) \\
(\mathrm{mag})\end{array}$ & $\begin{array}{c}M-T_{1} \\
(\mathrm{mag})\end{array}$ & $\begin{array}{c}\sigma\left(M-T_{1}\right) \\
(\mathrm{mag})\end{array}$ & $\begin{array}{c}T_{1}-T_{2} \\
(\mathrm{mag})\end{array}$ & $\begin{array}{c}\sigma\left(T_{1}-T_{2}\right) \\
(\mathrm{mag})\end{array}$ & $n$ \\
\hline GC1 & 234.11 & 1042.02 & 11.518 & 0.005 & 2.052 & 0.004 & 0.854 & 0.006 & 0.521 & 0.007 & 2 \\
\hline $\mathrm{GC} 2$ & 527.76 & 1440.62 & 11.569 & 0.001 & 2.103 & 0.002 & 0.875 & 0.001 & 0.533 & 0.001 & 2 \\
\hline GC3 & 536.95 & 828.35 & 11.437 & 0.006 & 2.075 & 0.004 & 0.872 & 0.001 & 0.514 & 0.002 & 2 \\
\hline GC4 & 923.12 & 205.26 & 11.311 & 0.004 & 2.149 & 0.002 & 0.885 & 0.005 & 0.529 & 0.009 & 2 \\
\hline GC5 & 1083.74 & 988.16 & 11.436 & 0.005 & 2.087 & 0.008 & 0.878 & 0.006 & 0.508 & 0.004 & 2 \\
\hline GC6 & 1088.02 & 1076.67 & 11.525 & 0.001 & 2.023 & 0.002 & 0.847 & 0.001 & 0.517 & 0.002 & 2 \\
\hline GC7 & 1748.78 & 552.49 & 11.242 & 0.006 & 2.138 & 0.010 & 0.883 & 0.006 & 0.525 & 0.007 & 2 \\
\hline GC8 & 1635.01 & 1124.44 & 11.368 & 0.004 & 2.125 & 0.004 & 0.887 & 0.004 & 0.520 & 0.004 & 1 \\
\hline GC9 & 648.74 & 1236.58 & 11.244 & 0.001 & 2.332 & 0.002 & 0.968 & 0.001 & 0.553 & 0.003 & 2 \\
\hline GC10 & 732.61 & 1112.18 & 11.740 & 0.001 & 1.646 & 0.002 & 0.749 & 0.001 & 0.492 & 0.001 & 2 \\
\hline GC11 & 1498.20 & 941.87 & 11.565 & 0.005 & 1.789 & 0.004 & 0.768 & 0.005 & 0.502 & 0.012 & 2 \\
\hline GC12 & 1810.99 & 1477.66 & 11.090 & 0.001 & 2.390 & 0.002 & 0.984 & 0.001 & 0.559 & 0.004 & 2 \\
\hline
\end{tabular}

$\bar{a}(X, Y)$ coordinates correspond to the reference system of Fig. 1. Magnitude and colour errors are the standard deviations of the mean, or the observed photometric errors for stars with one measurement. 


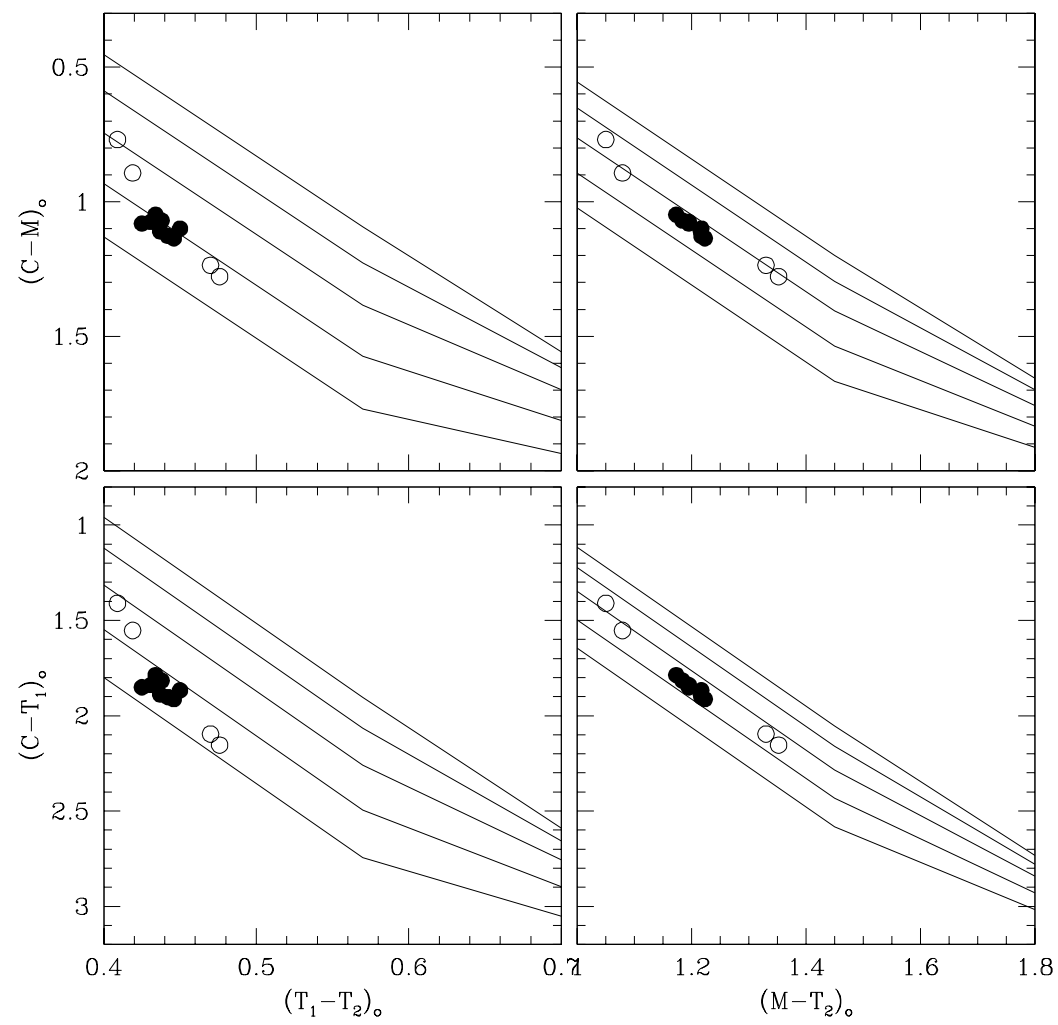

Figure 10. Unreddened Washington colour-colour diagrams for selected red giant candidates. Iso-abundance relations from Geisler et al. (1991) for 0.5 dex intervals from $[\mathrm{Fe} / \mathrm{H}]=-1.5$ to +0.5 are shown. Stars considered to be probable clump stars are represented with filled circles.

which is valid for stars with spectral types earlier than A2. However, the TO magnitude of NGC $2627\left(M_{V} \sim 1.2\right)$ suggests an MK type not earlier than A2. They assigned membership probabilities on the basis of the concordance in the evolutionary position in the $(V, B-V),(V, V-I)$ and $(U-B, B-V)$ planes and the allowance of deviations from cluster reddening and Walker's (1985) zero-age main sequence (ZAMS) of 0.12 and 0.4 mag, respectively. Ramsay \& Pollaco's fig. 18 shows that cluster members do not form any star sequence in the $(U-B, B-V)$ colour-colour diagram; instead, they form a group of stars confined to a small colour range that is difficult to fit properly by the ZAMS. For this reason, we conclude that the colour excess, distance and age derived by Ramsay \& Pollaco might not be reliable. On the other hand, Phelps et al. (1994) tabulated for the cluster $\delta V=1.6$, corresponding to a morphological age index (MAI) of 2.8 Gyr (Janes \& Phelps 1994), so that the cluster turns out to be twice as old as our estimated cluster age. At any rate, since they focused on a search for the oldest open clusters, they did not pay enough attention to this cluster. Consequently, our resulting fundamental parameters become a valuable contribution to the knowledge of NGC 2627.

The growing number of studies of intermediate-age clusters (IACs) of our Galaxy with well-determined fundamental parameters allows the investigation of their overall properties in the context of the chemical evolution of the Galactic disc. They define a specific epoch of such evolution, separated from the earliest epoch of the formation of the Galactic disc as well as from the more recent star formation events. Their age range is relatively quite narrow in comparison with the whole history of the Milky Way. For our purposes, we define here as IACs those clusters with ages between 0.6 and 2.5 Gyr. Note that IACs in the Large Magellanic Cloud are considered to be all the clusters in the age range 1-3 Gyr (Piatti et al. 2002; Geisler et al. 2003). We compiled a list of Galactic IACs from
Table 4. Washington abundance-sensitive indices.

\begin{tabular}{lrrrr}
\hline Star & \multicolumn{1}{c}{$\Delta_{1}^{\prime}$} & $\Delta_{3}^{\prime}$ & $\Delta_{4}^{\prime}$ & $\Delta_{5}^{\prime}$ \\
\hline GC1 & -0.007 & 0.030 & -0.069 & -0.064 \\
GC2 & -0.022 & 0.023 & -0.085 & -0.061 \\
GC3 & 0.011 & 0.067 & -0.078 & -0.064 \\
GC4 & 0.016 & 0.066 & -0.057 & -0.048 \\
GC5 & 0.028 & 0.121 & -0.073 & -0.052 \\
GC6 & -0.013 & 0.026 & -0.074 & -0.049 \\
GC7 & 0.019 & 0.072 & -0.058 & -0.047 \\
GC8 & 0.020 & 0.100 & -0.068 & -0.058 \\
GC9 & 0.025 & 0.110 & -0.111 & -0.088 \\
GC10 & -0.125 & -0.120 & -0.139 & -0.122 \\
GC11 & -0.112 & -0.102 & -0.087 & -0.084 \\
GC12 & 0.040 & 0.131 & -0.106 & -0.076 \\
\hline
\end{tabular}

a search in the WEBDA data base and the available literature. Table 5 presents the results of our search. The cluster distances, ages and metallicities come mainly from the new catalogue of open clusters built by Chen et al. (2003), although we also complemented such information for some clusters with results from recent individual studies.

Fig. 11 shows the distribution of the cluster sample projected on to the Galactic $(X, Y)$ coordinate plane, where $Y$ is defined to be positive in the direction of Galactic rotation and radially outward, respectively. The position of the Sun $\left(R_{\mathrm{GC}_{\odot}}=8.5 \mathrm{kpc}\right)$ is also represented in Fig. 11 with two perpendicular axes, while the curve corresponds to a Galactocentric radius of $R_{\mathrm{GC}}=10 \mathrm{kpc}$. Open circles and the filled square represent IACs taken from the literature and NGC 2627, respectively. Far from existing as a uniform distribution of clusters, they appear to have been observed through different lines of sight, as allowed by both the cluster density and/or 
Table 5. Intermediate-age open cluster fundamental parameters.

\begin{tabular}{|c|c|c|c|c|c|c|}
\hline Star cluster & $l(\mathrm{deg})$ & $\mathrm{b}(\mathrm{deg})$ & $d(\mathrm{pc})$ & Age (Gyr) & {$[\mathrm{Fe} / \mathrm{H}]$} & References $^{a}$ \\
\hline NGC 2360 & 229.80 & -1.43 & 1887 & 0.60 & -0.15 & 1 \\
\hline NGC 6811 & 79.44 & 11.95 & 1040 & 0.70 & 0.00 & 7 \\
\hline King 7 & 149.76 & -1.04 & 2200 & 0.70 & -0.40 & 8 \\
\hline NGC 2266 & 187.78 & 10.28 & 3400 & 0.63 & -0.26 & 9 \\
\hline NGC 5822 & 321.70 & 3.59 & 917 & 0.66 & -0.02 & 1 \\
\hline NGC 3960 & 294.36 & 6.18 & 2258 & 0.66 & -0.17 & 1 \\
\hline Haffner 6 & 227.85 & 0.25 & 3400 & 1.00 & 0.00 & 10 \\
\hline ESO 96-SC04 & 305.35 & -3.17 & 11800 & 0.70 & 0.00 & 11 \\
\hline NGC 2477 & 253.59 & -5.83 & 1222 & 0.70 & 0.01 & 1 \\
\hline Berkeley 33 & 225.43 & -4.62 & 4500 & 0.70 & -0.70 & 12 \\
\hline NGC 2355 & 203.30 & 11.80 & 2200 & 0.70 & -0.07 & 1 \\
\hline NGC 6940 & 69.90 & -7.14 & 770 & 0.72 & 0.01 & 1 \\
\hline NGC 2632 & 205.92 & 32.48 & 187 & 0.72 & 0.14 & 1 \\
\hline NGC 2423 & 230.48 & 3.54 & 766 & 0.73 & 0.14 & 1 \\
\hline Melotte 25 & 180.06 & -22.34 & 45 & 0.78 & 0.13 & 1,2 \\
\hline NGC 2204 & 226.01 & -16.10 & 2629 & 0.78 & -0.33 & 1 \\
\hline Berkeley 2 & 119.70 & -2.31 & 5250 & 0.79 & -0.40 & 13 \\
\hline Berkeley 104 & 117.63 & 1.22 & 4365 & 0.79 & 0.07 & 1 \\
\hline NGC 2304 & 197.20 & 8.89 & 3991 & 0.79 & -0.32 & 1 \\
\hline UKS 2 & 276.04 & -3.07 & 6800 & 0.80 & 0.00 & 14 \\
\hline Berkeley 23 & 192.60 & 5.44 & 6918 & 0.79 & 0.07 & 1 \\
\hline NGC 5823 & 321.16 & 2.46 & 1260 & 0.79 & -0.10 & 15 \\
\hline NGC 1901 & 279.05 & -33.64 & 450 & 0.60 & 0.00 & 16 \\
\hline Berkeley 69 & 174.42 & 11.79 & 2860 & 0.90 & -0.40 & 17 \\
\hline NGC 6134 & 334.91 & -0.19 & 913 & 0.92 & 0.18 & 1 \\
\hline Berkeley 64 & 131.91 & 4.60 & 3981 & 1.00 & -0.61 & 1 \\
\hline Tombaugh 1 & 232.22 & -7.32 & 2900 & 1.00 & 0.00 & 18 \\
\hline Pismis 19 & 314.68 & -0.38 & 2400 & 1.00 & -0.10 & 19 \\
\hline Berkeley 81 & 33.64 & -2.51 & 3000 & 1.00 & 0.00 & 20 \\
\hline NGC 2158 & 186.64 & 1.78 & 3600 & 2.00 & -0.23 & 1,3 \\
\hline Berkeley 29 & 197.98 & 8.02 & 14871 & 1.05 & -0.18 & 1 \\
\hline Berkeley 22 & 199.80 & -8.05 & 7663 & 1.06 & -0.30 & 1 \\
\hline Pismis 3 & 257.86 & 0.46 & 1190 & 2.00 & -0.40 & 21 \\
\hline NGC 2243 & 239.50 & -17.97 & 4458 & 1.07 & -0.44 & 1 \\
\hline NGC 2660 & 265.85 & -3.03 & 2826 & 1.07 & -0.18 & 1 \\
\hline NGC 2506 & 230.57 & 9.92 & 3460 & 1.10 & -0.37 & 1 \\
\hline NGC 2420 & 198.11 & 19.63 & 3085 & 1.11 & -0.26 & 1 \\
\hline King 11 & 117.16 & 6.47 & 2892 & 1.11 & -0.23 & 1 \\
\hline NGC 752 & 137.18 & -23.35 & 457 & 1.12 & -0.08 & 1 \\
\hline IC 4651 & 340.08 & -7.90 & 888 & 1.14 & 0.09 & 1 \\
\hline NGC 6208 & 333.75 & -5.76 & 939 & 1.17 & -0.03 & 1,4 \\
\hline NGC 3680 & 286.78 & 16.92 & 938 & 1.19 & -0.09 & 1 \\
\hline Pismis 18 & 308.18 & 0.26 & 2240 & 1.20 & 0.00 & 19 \\
\hline Collinder 74 & 198.98 & -10.40 & 2500 & 1.30 & 0.07 & 5 \\
\hline Collinder 110 & 209.65 & -1.98 & 1950 & 1.40 & 0.00 & 6 \\
\hline NGC 6819 & 73.97 & 8.48 & 2360 & 1.49 & 0.07 & 1 \\
\hline Berkeley 14 & 162.86 & 0.71 & 5495 & 1.60 & -0.32 & 5 \\
\hline NGC 2141 & 198.08 & -5.81 & 4033 & 1.70 & -0.26 & 1 \\
\hline NGC 7789 & 115.48 & -5.37 & 2337 & 1.71 & -0.08 & 1 \\
\hline NGC 7142 & 105.34 & 9.48 & 1686 & 1.88 & 0.04 & 1 \\
\hline NGC 7044 & 85.87 & -4.13 & 2960 & 1.60 & 0.00 & 20 \\
\hline Berkeley 31 & 206.25 & 5.12 & 8272 & 2.05 & -0.50 & 1 \\
\hline Berkeley 21 & 186.84 & -2.51 & 5000 & 2.18 & -0.83 & 1 \\
\hline NGC 6939 & 95.90 & 12.30 & 1185 & 2.21 & 0.02 & 1 \\
\hline
\end{tabular}

${ }^{a}$ References: (1) Chen et al. (2003); (2) Gratton (2000); (3) Carraro, Girardi \& Marigo (2002); (4) Piatti et al. (1995); (5) Ann et al (1999); (6) Dawson \& Ianna (1998); (7) Glushkova, Batyrshinova \& Ibragimov (1999); (8) Durgapal, Pandey \& Mohan (1997); (9) Kaluzny \& Mazur (1991); (10) Patat \& Carraro (1995); (11) Carraro, Vallenari \& Ortolani (1995); (12) Mazur, Kaluzny \& Krzeminski (1993); (13) Ann, Park \& Kang (1998); (14) Bica, Ortolani \& Barbuy (2000); (15) Janes (1981); (16) Pavani et al. (2001); (17) Pandey et al. (1997); (18) Carraro \& Patat (1995); (19) Piatti et al. (1998); (20) Sagar \& Griffiths (1998); (21) Carraro \& Ortolani (1994). 


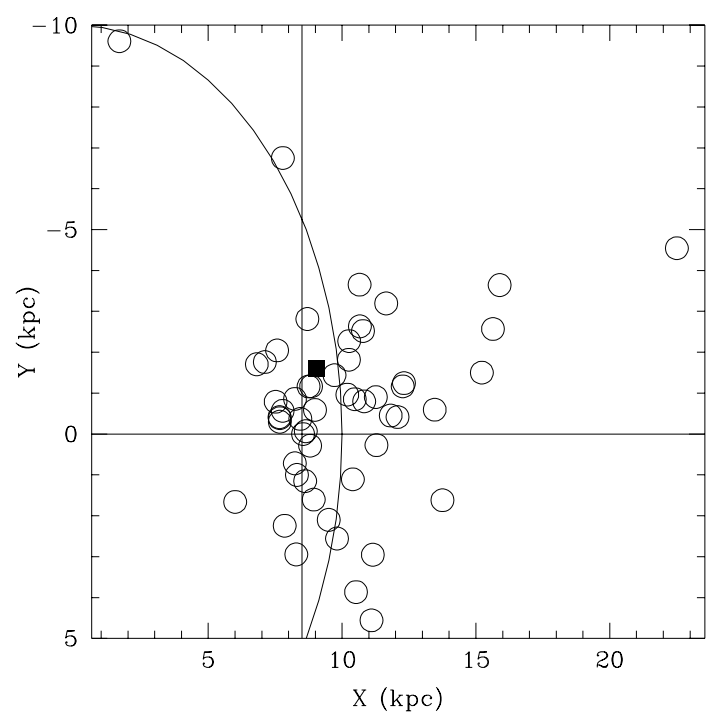

Figure 11. Galactic intermediate-age clusters of Table 5 plotted in the (X, $Y$ ) coordinate plane. The internal axes indicate the position of the Sun, while the curve corresponds to a circle of Galactocentric distance $R_{\mathrm{GC}}=10 \mathrm{kpc}$. Open circles and the filled square represent clusters of Table 5 and NGC 2627 , respectively.

the interstellar absorption. No conclusion can be drawn on their birthplaces since they could have travelled through the disc and have changed their relative positions (see e.g. Piatti, Clariá \& Abadi 1995). According to Fig. 12, IACs appear to have heights $(|Z|)$, with few exceptions, from the Galactic plane up to $0.5 \mathrm{kpc}$, which means that most of them were born and moved within the thin disc (Ojha et al. 1996; Larsen \& Humphreys 2003; Reddy et al. 2003). Indeed, to reach higher $|Z|$ values clusters should preferentially be born in the thick disc; otherwise, they would need high initial perpendicular velocities, which are unlikely for objects confined near the Galactic plane.

The sampled IACs do not show any trend between their metal abundances and ages (bottom panel in Fig. 12) but there is some

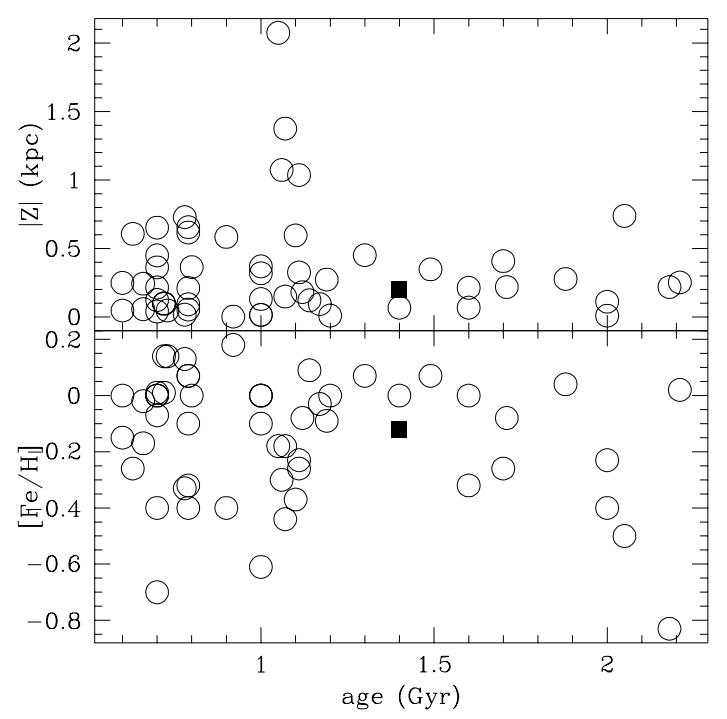

Figure 12. Relationships between cluster heights and metallicities as a function of cluster ages. Symbols are the same as in Fig. 11.

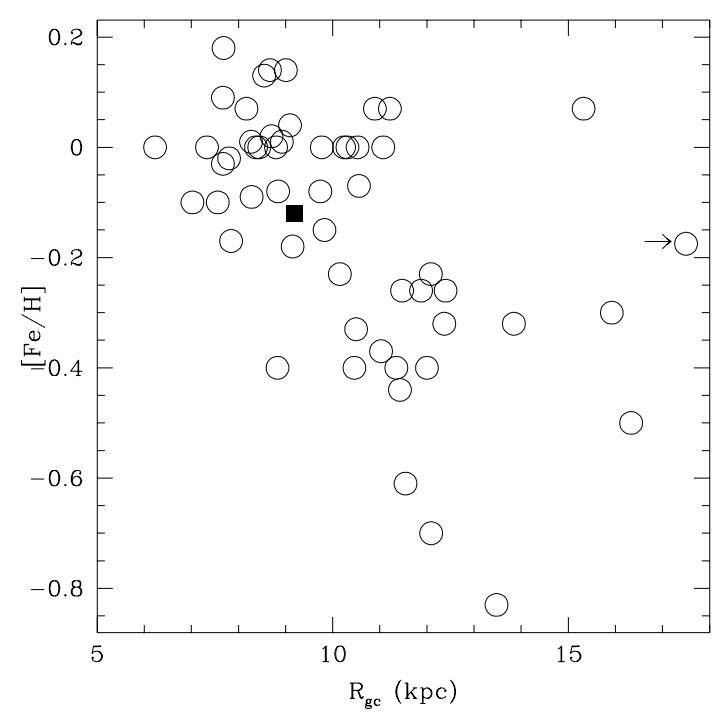

Figure 13. Metallicity versus Galactocentric distance for clusters of Table 5. Symbols are the same as in Fig. 11.

indication that they were born in different epochs more or less distinguishable from the disc lifetime, rather than in a continuous cluster formation process. Particularly, we identified peaks of cluster formation at $\sim 0.7-0.8,1.0-1.1,1.6-1.7$ and 2.0-2.1 Gyr, separated by relatively quiescent epochs of $\sim 0.2-0.3$ Gyr long. Their metallicities cover a wide range, from values as deficient in heavy elements as $[\mathrm{Fe} / \mathrm{H}] \sim-0.8$ until metal-rich ones of the order of $\sim+0.2$ dex. The spread in chemical compositions is caused by the dependence of cluster metallicities on their Galactocentric distance, as shown in Fig. 13. Note for example that clusters more metalpoor than $[\mathrm{Fe} / \mathrm{H}]=-0.2$ are on average located at Galactocentric distances $R_{\mathrm{GC}}>10 \mathrm{kpc}$. We estimated a formal metal abundance radial gradient of $-0.08 \pm 0.02$, in very good agreement with the most frequently derived value for the Galactic disc (see e.g. Piatti et al. 1995; Chen et al. 2003, and references therein). In this respect, from Fig. 13, we cannot rule out the alternative of a step function for the radial abundance distribution of open clusters (Twarog, Ashman \& Anthony-Twarog 1997; Corder \& Twarog 2001), although a more complete sample of clusters is needed to test such discontinuity.

Fig. 14 gives evidence that some scattered correlation exists between the Galactocentric distance $R_{\mathrm{GC}}$ and the height $|Z|$, in the sense that the higher a cluster is found, the more distant from the Galactic Centre it is located. For example, clusters with $|Z|>0.5 \mathrm{kpc}$ are located beyond $10 \mathrm{kpc}$ from the Galactic Centre. However, clusters can also be found far from the Galactic Centre and near or in the Galactic plane. In spite of such correlation, cluster metallicities do not seem to behave similarly with respect to $|Z|$. Once the $[\mathrm{Fe} / \mathrm{H}]$ ratios are corrected for the abundance radial gradient, a considerable dispersion only arises from the $[\mathrm{Fe} / \mathrm{H}]$ versus $|Z|$ diagram (bottom panel in Fig. 14) for IACs. This result has been previously suggested by Twarog et al. (1997) using a cluster sample spanning the whole open cluster age range. On the contrary, Piatti et al. (1995) and Chen et al. (2003), among others, have found vertical abundance gradients of the order of $\sim-0.30 \mathrm{dex} \mathrm{kpc}^{-1}$ using also young to old open clusters. The $[\mathrm{Fe} / \mathrm{H}]$ versus $|Z|$ relation of Fig. 14 shows that more or less metal-poor and metal-rich clusters are found at high or low distances from the Galactic plane. 


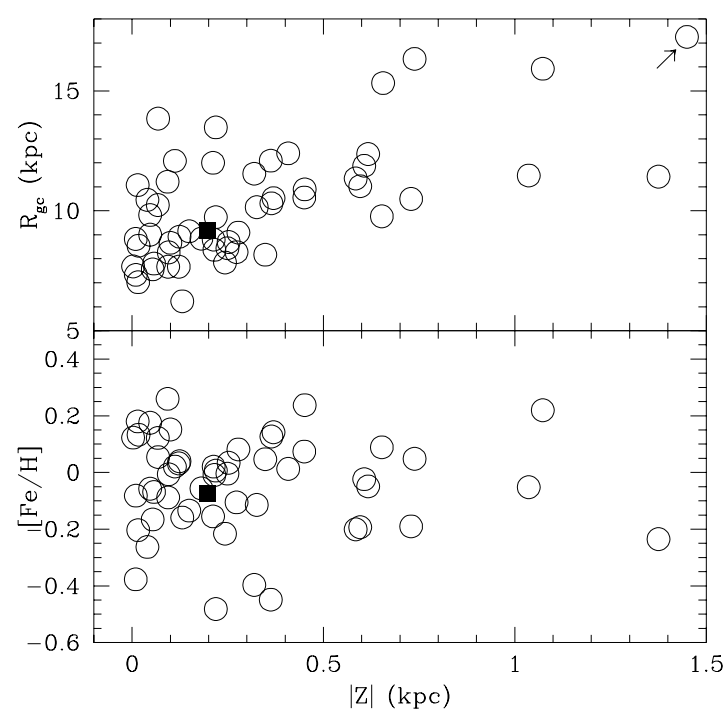

Figure 14. Relationships of cluster Galactocentric distances and metallicities as a function of cluster heights. Symbols are the same as in Fig. 11.

\section{CONCLUSIONS}

In this paper we present $C C D V I_{\mathrm{KC}}$ as well as CCD Washington system photometry in the field of the open cluster NGC 2627. The analysis of the photometric data leads to the following main conclusions:

(i) The observed $(V, V-I)$ diagram, corrected by field star contamination, reveals an upper main sequence (MS) with a remarkable hooking envelope, a dirty broad lower MS, and the presence of several giant clump stars. On the other hand, in the Washington $\left(T_{1}\right.$, $C-T_{1}$ ) diagram, the upper and lower MS envelopes appear to be much better defined and separated from the field stars, while the MS turnoff shows a smoother curvature. The cluster has a quite distinct secondary sequence in both CMDs, right above the single-star MS, a fact that could be put down to binary stars.

(ii) A metal abundance $[\mathrm{Fe} / \mathrm{H}]=-0.12 \pm 0.08$ relative to the Sun is estimated from the Washington system photometry of eight red giant clump stars.

(iii) Through the comparison of the cluster $(V, V-I)$ and $\left(T_{1}\right.$, $C-T_{1}$ ) CMDs with theoretical isochrones computed by the Geneva group, the following values for reddening, distance from the Sun and age were derived: $E(V-I)=0.25 \pm 0.05, E\left(C-T_{1}\right)=0.23 \pm$ $0.07,2.0 \pm 0.4 \mathrm{kpc}$ and $1.4 \mathrm{Gyr}$. The latter value agrees well with that derived from the $\delta V$ age index.

(iv) Using mainly the catalogue of open clusters recently published by Chen et al. (2003), we examined the fundamental properties of the open clusters with ages between 0.6 and $2.5 \mathrm{Gyr}$, including NGC 2627. We confirm the existence of a global age-metallicity relation for this cluster sample and we identified four peaks of cluster formation during this period, separated by relative quiescent epochs of $\sim 0.2-0.3$ Gyr. A wide range in metallicity is observed, which is clearly due to the dependence of cluster metallicity with Galactocentric distance. A radial abundance gradient $\partial[\mathrm{Fe} / \mathrm{H}] / \partial R_{\mathrm{GC}}=$ $-0.08 \pm 0.02$ is derived for this cluster sample, but no clear indication of an abundance $Z$ gradient is seen.

\section{ACKNOWLEDGMENTS}

We are gratefully indebted to the CTIO staff for their hospitality and support during the observing run. This work was partially sup- ported by the Argentinian institutions CONICET, SECYT (Universidad Nacional de Córdoba), Agencia Córdoba Ciencia and Agencia Nacional de Promoción Científica y Tecnológica (ANPCyT). This work is based on observations made at Cerro Tololo Inter-American Observatory, which is operated by AURA, Inc., under cooperative agreement with the NSF.

\section{REFERENCES}

Ahumada J. A., 1995, Rev. Mex. Astron. Astrofis., Ser. Conf., 8, 89 Ann H. B., Park Y. H., Kang Y. W., 1998, J. Korean Astron. Soc., 31, 39 Ann H. B., Lee M. G., Chun M. Y., Kim S. L., Jeon Y. B., Park B. G., 1999, J. Korean Astron. Soc., 32, 7

Bessell M. S., 1979, PASP, 91, 589

Bica E., Ortolani S., Barbuy B., 2000, A\&AS, 145, 399

Burstein D., Heiles C., 1982, AJ, 87, 1165

Canterna R., 1976, AJ, 81, 228

Carraro G., Ortolani S., 1994, A\&A, 291, 106

Carraro G., Patat F., 1995, MNRAS, 276, 563

Carraro G., Vallenari A., Ortolani S., 1995, A\&A, 300, 128

Carraro G., Girardi L., Marigo P., 2002, MNRAS, 332, 705

Chen L., Hou J. L., Wang J. J., 2003, AJ, 125, 1397

Clariá J. J., Mermilliod J.-C., Piatti A. E., Minniti D., 1994, A\&AS, 107, 39

Clariá J. J., Mermilliod J.-C., Piatti A. E., 1999, A\&AS, 134, 301

Clariá J. J., Piatti A. E., Lapasset E., Mermilliod J.-C., 2003, A\&A, 399, 543

Collinder P., 1931, Medd. Lunds. Astron. Obs. 2

Corder S., Twarog B.A., 2001, AJ, 122, 895

Cousins A. W. J., 1978, Mon. Not. Astron. Soc. S. Afr., 37, 62

Dawson D. W., Ianna P. A., 1998, AJ, 115, 1076

Dias W. S, Alessi B. S., Moitinho A., Lépine J. R., 2002, A\&A, 389, 871

Durgapal A. K., Pandey A. K., Mohan V., 1997, Bull. Astron. Soc. India, 25, 489

Dutra C. M., Bica E., 2000, A\&A, 359, 347

Friel E. D., Janes K. A., 1993, A\&A, 267, 75

Geisler D., 1996, AJ, 111, 480

Geisler D., Clariá J. J., Minniti D., 1991, AJ, 102, 1836 (GCM91)

Geisler D., Lee M. G., Kim E., 1996, AJ, 111, 1529

Geisler D., Piatti A. E., Bica E., Clariá J. J., 2003, MNRAS, 341, 771

Glushkova E. V., Batyrshinova V. M., Ibragimov M. A., 1999, Astron. Lett., 25,86

Gratton R., 2000, in Pallavicini R., Micela G., Sciortino S., eds, ASP Conf. Ser. Vol. 198, Stellar Clusters and Associations: Convection, Rotation, and Dynamos. Astron. Soc. Pac., San Francisco, p. 225

Janes K. A., 1981, AJ, 86, 1210

Janes K. A., Phelps R. L., 1994, AJ, 108, 1773

Kaluzny J., Mazur B., 1991, Acta Astron., 41, 191

King I. R., 1964, R. Obs. Bull., 82, 106

Landolt A. U., 1992, AJ, 104, 340

Larsen J. A., Humphreys R. M., 2003, AJ, 125, 1958

Lauberts A., 1982, The ESO/Uppsala Survey of the ESO(B) Atlas. European Southern Observatory, Garching

Lejeune T., Schaerer D., 2001, A\&A, 366, 538 (LS01)

Lyngå G., 1987, Catalogue of Open Cluster Data. Centre de Données Stellaires, Strasbourg

Mazur B., Kaluzny J., Krzeminski W., 1993, MNRAS, 265, 405

Melotte P. J., 1915, Mem. R. Astron. Soc., 60, 175

Mermilliod J.-C., Mayor M., 1989, A\&A, 219, 125

Mermilliod J.-C., Mayor M., 1990, A\&A, 237, 71

Mermilliod J.-C., Clariá J. J., Andersen J., Piatti A. E., 2001, A\&A, 375, 30

Meynet G., Mermilliod J.-C, Maeder A., 1993, A\&A, 9, 477

Ojha D. K., Bienayme O., Robin A. C., Creze M., Mohan V., 1996, A\&A, 311,456

Pandey A. K., Durgapal A. K., Bhatt B. C., Mohan V., Mahra H. S., 1997, A\&AS, 122, 111

Patat F., Carraro G., 1995, MNRAS, 272, 507

Pavani D. B., Bica E., Dutra C. M., Dottori H., Santiago B. X., Carranza G., Díaz R. J., 2001, A\&A, 374, 554 
Phelps R. L., Janes K. A., Montgomery K. A., 1994, AJ, 107, 1079

Piatti A. E., Clariá J. J., Abadi M. G., 1995, AJ, 110, 2813

Piatti A. E., Clariá J. J., Bica E., Geisler D., Minniti D., 1998, AJ, 116, 801

Piatti A. E., Sarajedini A., Geisler D., Bica E., Clariá J. J., 2002, MNRAS, 329, 556

Piatti A. E., Clariá J. J., Ahumada A. V., 2003, MNRAS, 340, 1249 (Paper I)

Ramsay G., Pollaco D. L., 1992, A\&AS, 94, 73

Reddy B. E., Tomkin J., Lambert D. L., Allende Prieto C., 2003, MNRAS, 340,304
Ruprecht J., 1966, Bull. Astron. Inst. Czech., 17, 33

Sagar R., Griffiths W. K., 1998, MNRAS, 299, 1

Stetson P. B., 1987, PASP, 99, 191

Trumpler R. J., 1930, Lick Obs. Bull., 14, 154

Twarog B. A., Ashman K. A., Anthony-Twarog B. J., 1997, AJ, 114, 2556

van den Bergh S., Hagen G. L., 1975, AJ, 80, 11

Walker A. R., 1985, MNRAS, 213, 889

This paper has been typeset from a $\mathrm{T}_{\mathrm{E}} \mathrm{X} / \mathrm{L} \mathrm{T}_{\mathrm{E}} \mathrm{X}$ file prepared by the author. 